\title{
Marked pointing facilitates learning part names: A test of lexical constraint versus social pragmatic accounts of word learning
}

\author{
Harumi KOBAYASHI ${ }^{1, *}$ (D), Tetsuya YASUDA ${ }^{1}$ and Ulf LISZKOWSKI ${ }^{2}$ \\ ${ }^{1}$ Tokyo Denki University, Japan \\ ${ }^{2}$ University of Hamburg, Germany \\ ${ }^{*}$ Corresponding author: Harumi Kobayashi, School of Science and Engineering, Tokyo Denki University, \\ Ishizaka Hatoyama-machi Hiki-gun, Saitama, 350-0394, Japan. Email: h-koba@mail.dendai.ac.jp
}

(Received 16 September 2020; revised 12 August 2021; accepted 03 December 2021)

\begin{abstract}
The empirical study of word learning is driven by a theoretical debate between lexical constraint and social-pragmatic accounts; it has still not been determined which of these two best explains the evidence. We investigated whether the markedness of a pointing accompanying a verbal reference could help to learn a part name. Participants were 35 two-and-ahalf-year-olds, 42 four-and-a-half-year-olds, and 38 undergraduate university students in Japan. The experimenter pointed to a novel part (embedded in a novel whole object) with either "marked" pointing, which was touching the part with a small circular motion, or with usual pointing. Touch accompanied by circular motion reliably elicited learning of part names in all age groups. Usual distal pointing without motion reliably elicited learning of whole object names. The pattern of findings rejects a whole-object constraint in early word learning and demonstrates that marked pointing can promote learning novel part names, supporting a social-pragmatic account.
\end{abstract}

Keywords: social-pragmatic account; word learning; markedness; pointing gesture; part names

\section{Introduction}

A challenge in word learning is that the relation between a word and what it denotes is not readily observable. Thus, the intended meaning cannot simply be learned by association; it must be inferred somehow (Quine \& Van, 1960; Sperber \& Wilson, 1995). Contemporary research has investigated how word learners know the relationships between words and their referents - that is, how they interpret the referential meaning of words (e.g., Bloom, 2002; Markman, 1991; Pinker, 2003; Tomasello, 2008).

Two major and distinct theoretical accounts that address this core issue of referential interpretation in word learning are the lexical constraint account and the social-pragmatic account. The lexical constraint account proposes that word learners are naturally equipped with lexical assumptions that constrain the possible meanings of given words. 
Two of the most important learning constraints are whole object and mutual exclusivity (Markman, 1991; Markman \& Wachtel, 1988). The whole object assumption states that when a word is used for an object, the learner thinks that this word refers to the whole object rather than to any of its parts or substance. The assumption of mutual exclusivity states that the learner assumes that only one word can refer to one referent because two object categories must be mutually exclusive. Au and Glusman (1990) reported that adults as well as children resisted assigning two novel names to the same object when there was no additional information, such as a hierarchy of names or names from different languages. Similarly, Merriman, Bowman, and MacWhinney (1989) claimed that mutual exclusivity is a default option that both children and adults use to integrate new and old words in their vocabulary. Some researchers have also suggested that these languagespecific biases, rather than being innate, may initially derive from domain-general statistical learning of associative patterns and regularities (Smith, 2001; Yu \& Smith, 2012).

In contrast, social-pragmatic accounts propose that words are learned by inferring speakers' referential intentions within a joint social-constructive process (Bruner, 1981; Clark, 1997, 2009; Tomasello, 2000, 2008; Tomasello \& Haberl, 2003). Rather than being equipped with language-specific adaptations for making initial lexical assumptions, the learner uses the speakers' pragmatic gestures or cues such as pointing, directional eye gaze, facial expression, or conversational contexts to make inferences about the speaker's intended use of words. For example, children consider the attentional focus of a speaker when learning word labels for objects (Baldwin, 1991); they distinguish accidental from intentional labeling contexts, which suggests against simple associations (Diesendruck, Markson, Akhtar \& Reudor, 2004). They learn labels not only for visible objects but also for absent objects and even for actions, depending on the speaker's intention as determined by shared experience (Akhtar, Carpenter \& Tomasello, 1996). Further, the learning does not appear to be language-specific as children also apply it when, for example, learning about facts rather than words (Diesendruck \& Markson, 2001).

Indeed, subsequent research has shown that infants understand speakers' referential intentions at an age when word learning is only beginning, even outside word learning situations, to determine what others communicate about with them (e.g., Behne, Liszkowski, Carpenter \& Tomasello, 2012; Gliga \& Csibra, 2009; Rüther \& Liszkowski, 2020). For example, one-year-olds are able to determine the referent of an ambiguous request that an adult directs at an array of three objects on the basis of what they have or have not previously experienced with the adults (Moll, Koring, Carpenter \& Tomasello, 2006; Tomasello \& Haberl, 2003; see also Saylor, Sabbagh \& Baldwin, 2002 for three- to fouryear-olds), and they are able to determine whether an exciting exclamation refers to a whole object in sight or to a specific aspect of that object depending on the utterer's intention as determined by what she has or has not seen (Moll et al., 2006).

While some researchers have subsequently suggested that social-pragmatic accounts sufficiently and more parsimoniously explain word learning compared to lexical accounts (e.g., Carston, 2008; Tomasello, 2008, 2009), other researchers have suggested that socialpragmatic cues may interact or refine lexical constraints. The latter idea has been investigated with conflicting cue paradigms to directly test what conditions are necessary for different accounts to make the best predictions. For example, Jaswal and Hansen (2006) asked young children to choose referent objects when pragmatic information of eye gaze and pointing conflicted with mutual exclusivity. They reported that children disregarded pragmatic cues and followed mutual exclusivity. Jaswal (2010) further examined children's tendency to relate a novel label to a novel object in a familiar-novel object pair in the presence of pragmatic cues of pointing and gazing at objects. They 
reported that children use mutual exclusivity to disambiguate speakers' meaning but that a combination of pragmatic cues may help override the notion that one object has only one label. However, in contrast to these conclusions, Grassmann, Stracke, and Tomasello (2009) found that when two-year-olds shared the common experience of interacting with an object and an adult, and the adult later excitedly uttered a novel label, children did not expect it to refer to the already commonly known object. Instead, they searched for an alternative referent. Grassmann et al. concluded that children did not use "purely" lexical principles; rather, they treated novel labels and objects in a pragmatic manner considering the speaker's intention.

An important test case is children's learning of part names. Learning part names should be challenging for young children if they purely follow a whole-object assumption. However, in their seminal study, Markman and Wachtel (1988) claimed that by combining the whole object and mutual exclusivity assumptions, children can also learn the names of object parts. With three-year-old participants, Markman and Wachtel showed that in an unfamiliar-object condition, children simply use the whole-object constraint, mistakenly thinking that the whole object (lung) is a "trachea." However, in a familiarobject (fish) condition, children use mutual exclusivity to correctly learn the name "dorsal fin," referring to part of the familiar object. On the other hand, social-pragmatic cues may also provide context to overcome possible whole-object constraints, as social-pragmatic information conveyed through gaze and gesture is an important source for word learning (e.g., Clark, 2009; Kita, 2003; Tomasello, 2008).

Hansen and Markman (2009) directly tested the whole-object principle and an effect of specific pointing gesture in part name learning with two- and three-year-olds. They taught children part names using a social-pragmatic gesture of tracing the contour of the object part. In the familiar whole-object condition, they showed a baseball cap and traced the contour of its brim (a novel name), saying, "This is a brim. See this brim." In the test, the experimenter placed the cap on the table so that the critical brim part was facing away from the child. The experimenter then asked the child, "Can you put your finger on the brim?" Their idea was that if children put their finger on the far part of the object, or if they moved so that they could more easily point at the part, then it could be concluded that children referred to the object part. Participants were also asked the same question in a novel whole-object (tea infuser) and a novel part-object (globe-shaped metal mesh) condition. Results from Hansen and Markman (2009) clearly supported the lexical constraint account of part-word learning over the social-pragmatic account. That is, study participants correctly identified novel part labels only in the known whole-object and novel part (baseball cap and brim) condition. This condition was intended to be the "mutual exclusivity is available" condition because children could use mutual exclusivity to discern the meaning of the novel word. However, in a condition with an unknown whole object and novel part, children did not respond correctly. If children mainly used the social-pragmatic gesture of tracing the part, they should have learned the novel part name in the unfamiliar object condition because the tracing gesture was presented in all experimental trials. Hansen and Markman concluded that children learned novel names for object parts only when mutual exclusivity could be used: "Mutual exclusivity is indispensable even when part labeling is accompanied by naturalistic communicative gestures" (2009, p. 592).

However, in Hansen and Markman's (2009) study, the possible effect of the socialpragmatic gesture may have been masked by their design because the whole-object response was easier to make than the part-object response. In their experiment, the target object part (brim) was always placed facing away from the child to ensure that the child 
made a part response. Consequently, this procedure meant that even when children randomly pointed at or touched the nearby object, their responses would be counted as a whole response. Thus, the two choices of the whole and the part were not equally accessible for participating children; rather, there was a design bias toward whole object responses.

The current study examined whether social-pragmatic gestures could help young children learn the names for novel parts of novel objects. By design, therefore, an assumption of mutual exclusivity could not be used regarding learning the part label. If social-pragmatic gesture alone could lead children to learn the names of unfamiliar parts of unfamiliar objects, this would speak against rigid lexical constraint accounts and instead suggest that children make flexible inferences about the gesturer's referential intentions. In order to assure that participants could not use mutual exclusivity, all whole objects and target-object parts were unfamiliar to children.

As a social-pragmatic gesture, we focused on pointing because it is an essential gesture in language development and human communication (Clark, 2009; Kita, 2003; Tomasello, 2008) and an important source of joint attention in word learning (e.g., Hansen \& Markman, 2009; Kobayashi, 1997, 1998; Kobayashi \& Yasuda, 2012; Mervis, Golinkoff \& Bertrand, 1994). Experimental evidence has revealed that one-year-olds comprehend and express the communicative, referential, and social intentions of pointing and can infer the referent of pointing acts in a variety of situations when the referent is a new, known, hidden, or sometimes even an absent object depending on the shared context (for an overview, see Liszkowski, 2018). Beyond shared context and common ground, the meaning of pointing gestures also derives from their accompanying characteristics (Liszkowski, 2014). For example, infants distinctly mark their social intentions to either obtain an object or help others find it through distinct intonation of accompanying vocalizations and gesture shape (Grünloh \& Liszkowski, 2015). Further, they use adults' distinct marking to make appropriate inferences about adults' social intentions (EsteveGibert, Prieto \& Liszkowski, 2017). Liebal, Carpenter, and Tomasello (2011) showed that such "markedness" of pointing also modulates two- and three-year-olds' referential interpretation of pointing. When children were presented with a big object (box) with a small "aspect" (e.g., a sticker attached to the back side of the box) and the experimenter then pointed in a "marked" way with repeated motion and exaggerated facial expression and intonation, children chose the aspect more often than when the experimenter used "unmarked" still pointing. Therefore, accompanying or marking characteristics of pointing may provide children with an additional source of information in interpreting a speaker's referential intention of a word label.

We adopted materials and procedures from Kobayashi (1998). Kobayashi showed that when the function (e.g., turning the nut) of the object part (nut) was demonstrated in addition to pointing at the part, two-year-old children could learn the names for object parts. In a test trial, when confronted with the label and a choice of the whole object or the separated part of the object, children systematically chose the part object. Hansen and Markman (2009) noted that, in Kobayashi's study, the children might have had difficulty recognizing the part (nut) that was separated from the whole object (U-shaped bolt). This seems a reasonable criticism; however, half of Kobayashi's trials included "transfer" trials in which the target part appeared on another whole object with a different shape (I-shaped bolt). Kobayashi (1998) reported that there was no difference in the frequency of part objects chosen between the isolated part set and the transferred part set, supporting the interpretation that children had learned the part label. 
In the current study, participants were presented with an object having an identifiable additional part and then heard a spoken label. In addition, the label was accompanied by different types of pointing gestures. In our main test, the "isolated trials," the whole object and the part object were separately presented, and participants were asked to choose whether the label referred to the whole or part object. In additional adjacent trial ("transfer trial"), to confirm the child's interpretation as a part name, participants were then presented with a choice of the same part object on a different whole object and the previous whole object. Our main question was how participants would interpret the reference in the first trial type, the "isolated trials." In a pure lexical constraint account, in the "isolated trials," participants should take the word label to denote the whole object, following the whole object constraint. Instead, in a social-pragmatic account, participants should be influenced by the labeler's intention conveyed through distinct pointing gestures. We then tested participants with a supposedly more difficult "transfer test" in which the participants needed to recognize that the learned object part can be attached to other whole objects.

We used two types of social-pragmatic cues to mark the intended part-whole choice of reference: distance and motion. The point either touched the object part or was $7 \mathrm{~cm}$ away; it was also either accompanied by a small circular motion of the index finger or not accompanied by such a motion. Our prediction was that if markedness of the pointing gestures helps learn a part label, a shorter distance of pointing should produce more part responses. We reasoned that touch-pointing requires more effort than distant pointing because it requires moving one's arm closer to the referent, which results in an exaggerated and thus marked form of attention-directing. We also predicted that adding a circular motion to the pointing finger will produce more part responses, again, because it requires more effort than still pointing and should thus mark some specific aspect. We reasoned that the combination of touch and motion could be especially helpful for the youngest age group to learn part names. To see whether these effects would be present in children, we first tested adults to check for the predicted effects, and then we tested fourand-a-half-year-olds who should exhibit flexibility in word learning as well as a more contested younger age group of two-and-a-half-year-olds, for whom word learning has been suggested to be more strongly constrained by mutual exclusivity (Gollek \& Doherty, 2016; Jaswal \& Hansen, 2006; Kalashnikova, Mattock \& Monaghan, 2016). If children rely on social-pragmatic cues from the beginning, then the youngest children should already show sensitivity to marked pointing and learn labels for parts of objects in the "isolated trials" showing no difference from the "transfer trials." The case of distant pointing without motion presents a kind of "unmarked" pointing cue. Because the distant still pointing cue does not reveal a clear intention to refer to a specific aspect or part of an object, participants could be expected to interpret it as referring to the whole object. Thus, for the "unmarked" distal pointing cue in "isolated trials," there was no empirical distinction between lexical constraints and social-pragmatic accounts.

\section{Method}

\subsection{Participants}

Participants were 35 two-and-a-half-year-old children $(\mathrm{M}$ month $=30.91, \mathrm{SD}=4.58$, range $=24-40 ; 18$ boys $)$ and 42 four-and-a-half-year-old children ( $\mathrm{M}$ month $=54.19$, $\mathrm{SD}=3.93$, range $=48-62 ; 21$ boys) from two preschools in Yokohama and Saitama, Japan. The data of three additional two-and-a-half-year-old children (two boys) were excluded 
from data analysis because they did not complete the task (one child was fussy; two children did not comply with the task). For practical reasons, data collection was terminated because there were no more participants available at the preschools. The children were from middle-class families. Their preschool teachers reported no delays of cognitive or language development. Before and during the warm-up period, we interacted naturally with the children and did not detect any language delays. The adult participants were 38 undergraduate university students in Saitama, Japan (M year $=20.50, S D=1.11$, range $=19-24,17$ males). Sample sizes were estimated based on conventional sizes reported in the literature as well as practical constraints imposed by time and access to data collection. The experiment was conducted in accordance with the university's code of confidentiality and ethical treatment of human subjects.

\subsection{Materials}

Stimuli consisted of four sets of approximately similar sizes used in Kobayashi (1998) and Kobayashi (2002, 2007): a nut set (object part: nut; whole object: U-shaped bolt; Figure 1a), a plate-hanger set (object part: spring; whole object: wire similar to platehanger; Figure 1b), a pump set (object part: pump; whole object: bottle of an unfamiliar shape; Figure 1c), and a letter-scale set (object part: clip; whole object: metal-body; Figure 1d).

The test items in the first test phase (an isolated-part trial) consisted of the part choice object (e.g., the nut) and the whole choice object (e.g., the U-shaped bolt without the nut). The test items for the second test phase (a transfer trial) consisted of part choice objects with distinct shapes on a different whole object (e.g., an I-shaped bolt with a nut) and the previous whole choice object without the part (e.g., a U-shaped bolt without the nut).

\section{Traning phase}

a

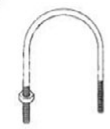

b

b

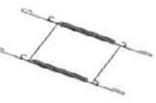

(C)

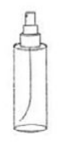

d

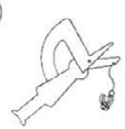

(P)

Isolated
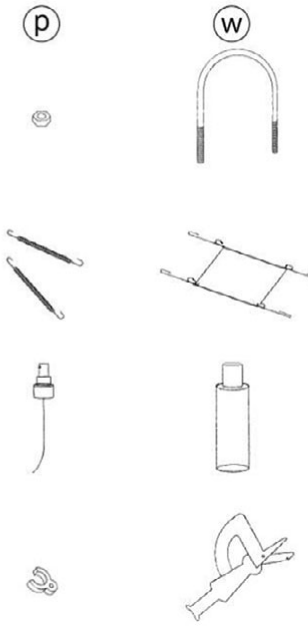

Test phase

Transfer
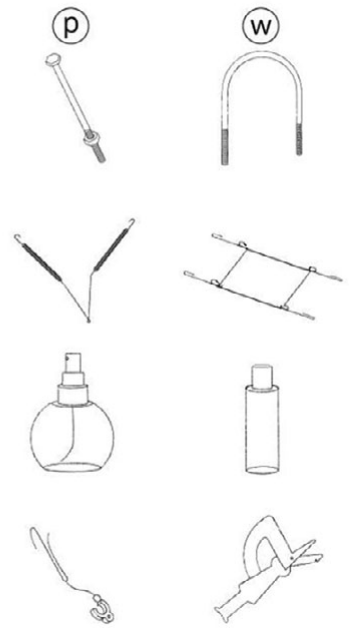

Figure 1. Stimulus sets a-d. $p$ denotes part choice; $w$ denotes whole choice. 
Our previous study reported that Japanese four-year-olds $(\mathrm{N}=20)$ did not know the whole or part items used in the current experiment or their names (Kobayashi, 2002).

\subsection{Procedures}

Participants were randomly assigned to one of four conditions resulting from crossing two factors with two levels: distance $(0 \mathrm{~cm}$ : touch; $7 \mathrm{~cm}$ : distant) and motion (still, circular). In the touch conditions, the extended index finger touched the part object. In the distant conditions, the index finger was $7 \mathrm{~cm}$ away from the object part. In the circular motion conditions, the index finger moved $1 \mathrm{~cm}$ in diameter; in the still conditions, the index finger did not move (Figure 2).

In the labeling phase, the experimenter first placed the connected standard set (e.g., a U-shaped bolt with a nut) on a white paper plate. The experimenter then pointed according to one of the four conditions while saying in Japanese "kore (this) wa (particle) natto (noun: a part name) desu (verb: is)" (“This is (a) nut") twice. Following the labeling phase, the isolated-part test began. The experimenter presented the two test items disconnected on separate paper plates. The experimenter then asked the child, "Docchi (which) no (particle) osara (plate) ni (particle) natto (noun: a part name) ga (particle) aru (verb) kana (particle)?” (“On which plate do you find (a) nut?"). After the isolated-part test, the transfer test began. The experimenter presented the two transfer test items (i.e., the object part on a different whole object and the whole object of the previous isolated trial) in the same manner and asked, "On which plate do you find (a) nut?" The procedures were identical for each object set, but each set used different target labels. Thus, there were four object sets, and for each object set, there was one isolated-part test and one transfer test, resulting in four isolated-part tests and four transfer tests, totaling eight trials for each
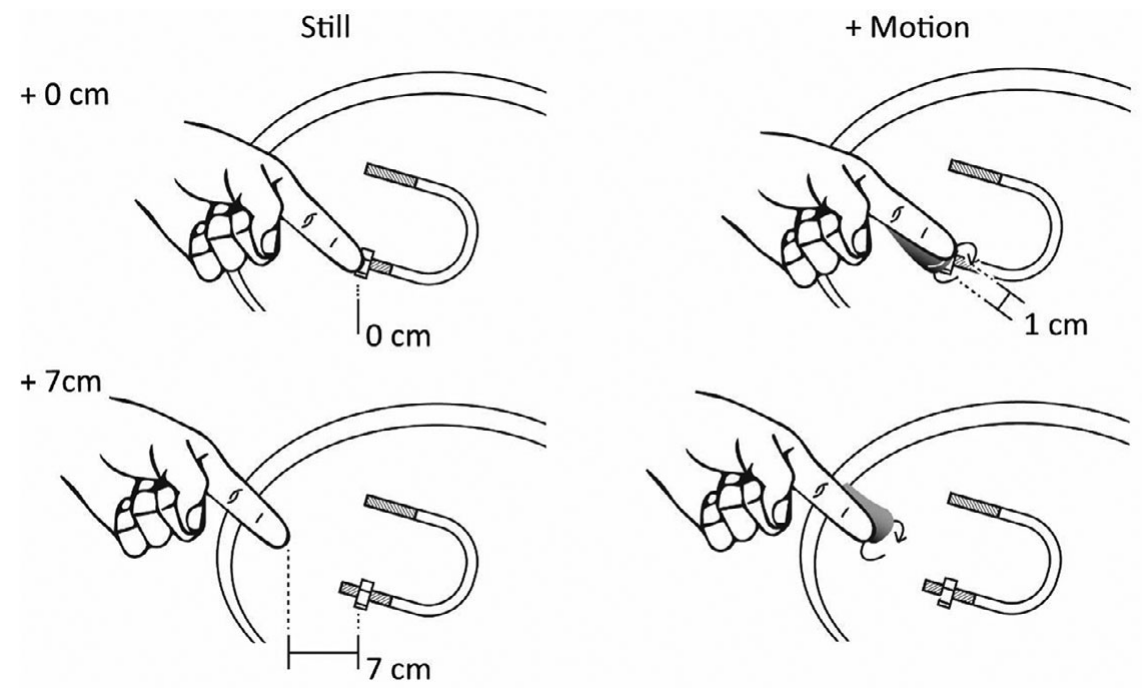

Figure 2. Still pointing and pointing with motion in the part name labeling phrase. The experimenter pointed at the novel part (nut) either with still pointing or pointing with a small circular motion over the part. The experimenter also touched the part with the index-finger or did not touch it. 
participant. For the university students, the same materials and procedures were used as for the children, except that we taught nonsense words as part names in a foreign language to the adult participants so as to control for their prior word knowledge.

\subsection{Scoring and analyses}

The isolated-part test and the transfer test were scored independently. Each score was calculated based on the participant's performance across all four trials. Participants received one point when they correctly selected the object part. Thus, in each of the four conditions, scores for each of the two tests ranged from 0 to 4 .

In the first step, we submitted the data to an omnibus ANOVA including all factors to reveal the pattern of mean performance across age (three levels), cue distance (two levels), cue motion (two levels), and trial type (two levels). We then analyzed each age group separately. In each analysis, we tested whether the effects would be similar in isolated trials and transfer trials, as predicted by the social-pragmatic account.

In the second step, complementary analysis, we tested whether part-label learning exceeded chance level and compared the averaged binary performance across trials against the expected chance value given a random choice. In the third step, final analysis, we explored whether there was an indication that participants switched their answers, perhaps as a result of pragmatic misunderstanding. Responses were classified into two types: absence-of-change (both trials "whole" response or both trials "part" response) and presence-of-change (change from "whole" response to "part" response or vice versa). Difference scores were then calculated (i.e., (number of absence-of-change - number of presence-of-change) / (number of absence-of-change + number of presence-of-change)). Positive values (i.e., $0 \leq$ difference score $\leq 1.0$ ) indicated no systematic change of choice, and negative values (i.e., $-1.0 \leq$ difference score $<0$ ) indicated change of choice. For each age group, we then planned a one-sample t-test against chance set at 0 .

\section{Results}

\subsection{Performance across conditions}

Figure 3 shows a jittered boxplot of part choices for each age group, distance, motion, and trial type. An initial omnibus ANOVA with the factors 3 (Age: two-and-a-half-year-olds, four-and-a-half-year-olds, adult) $\times 2$ (Distance: touch, distant) $\times 2$ (Motion: still, circular) $\times 2$ (Trial type: isolated, transfer) on object-part response scores as the dependent measure using R (x64 3.5.1), with Age, Distance, and Motion as betweenparticipants variables and Trial type as a within-participants variable revealed significant main effects of Age $\left(F_{2,103}=5.292, p<.01, \eta^{2}=0.032\right)$, Distance $\left(F_{1,103}=114.642, p<\right.$ $\left..001, \eta^{2}=0.349\right)$, and Motion $\left(F_{1,103}=27.432, p<.001, \eta^{2}=0.084\right)$. These main effects were qualified by a number of significant interactions: Age $\times$ Distance $\left(F_{2,103}=14.067\right.$, $p<.001, \eta^{2}=0.086$. $)$, Age $\times$ Motion $\left(F_{2,103}=3.916, p<.05, \eta^{2}=0.024\right)$, Distance $\times$ Motion $\left(F_{1,103}=4.832, p<.05, \eta^{2}=0.015\right)$, and Distance $\times$ Trial type $\left(F_{1,103}=4.996\right.$, $\left.p<.05, \eta^{2}=0.004\right)$. No other effects were significant.

Visual inspection of Figure 3 also suggests that there is a developmental change in the distribution of responses. An age-related difference is clear in the touch condition but not in the distance condition. Given that age interacted with our manipulated variables and our theoretical interest in each age group, we subsequently ran the analyses separately for each age group. 


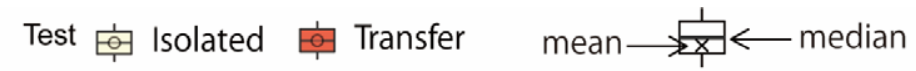

touch $(0 \mathrm{~cm})$ distant $(+7 \mathrm{~cm})$ adults

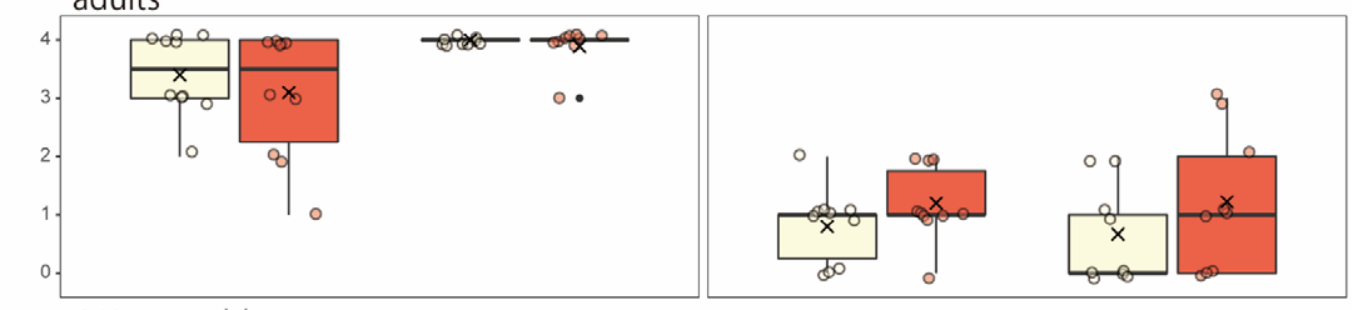

$4 \frac{1}{2}$ year-olds

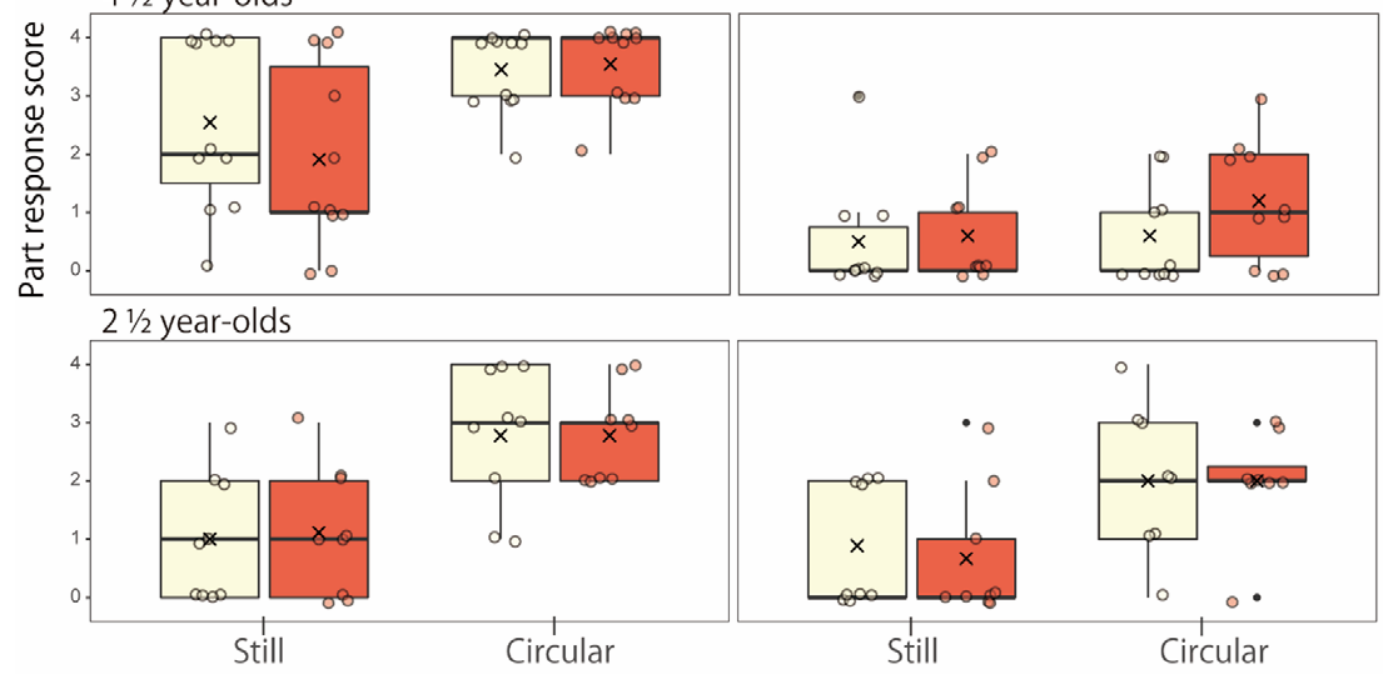

Figure 3. Jittered boxplot of part response scores for each age group and pointing. 


\section{Adults}

Regarding adults, a 2 (Distance) $\times 2$ (Motion) $\times 2$ (Trial type) ANOVA revealed a large main effect of Distance $\left(F_{1,34}=122.832, p<.001, \eta^{2}=0.730\right)$, with more part name learning for touching $(\mathrm{M}=3.58, \mathrm{SE}=0.17)$ than $7 \mathrm{~cm}$ distance $(\mathrm{M}=0.97, \mathrm{SE}=0.19)$. Motion produced no additional significant effects. There was a significant but fairly small interaction effect between Distance and Trial type $\left(F_{1,34}=15.649, p<.001, \eta^{2}=0.012\right)$. Resolving the interaction through direct comparisons, however, confirmed that, for both isolated and transfer trials, touch-pointing yielded more part-name learning than the $7 \mathrm{~cm}$ distance (isolated trials, touch: $\mathrm{M}=3.68, \mathrm{SE}=0.14 ; 7 \mathrm{~cm}: \mathrm{M}=0.74, \mathrm{SE}=0.22, p<.001$; transfer trials, touch: $\mathrm{M}=3.47, \mathrm{SE}=0.21 ; 7 \mathrm{~cm}: \mathrm{M}=1.21, \mathrm{SE}=0.26, p<.001)$.

\section{Four-and-a-half-year-olds}

Regarding four-and-a-half-year-olds, a 2 (distance) $\times 2$ (motion) $\times 2$ (trial type) ANOVA again revealed a significant main effect of Distance $\left(F_{1,38}=50.851, p<.001\right.$, $\left.\eta^{2}=0.465\right)$, with more part-name learning for touching $(\mathrm{M}=2.86, \mathrm{SE}=0.27)$ than $7 \mathrm{~cm}$ distance $(M=0.73, \mathrm{SE}=0.18)$. There was also a main effect for Motion $\left(F_{1,38}=7.319\right.$, $\left.p<.05, \eta^{2}=0.067\right)$, with more part-name learning for circular motion $(\mathrm{M}=2.26, \mathrm{SE}=$ $0.33)$ than still pointing $(\mathrm{M}=1.43, \mathrm{SE}=0.32)$.

Again, there was a small interaction effect of Trial type and Distance $\left(F_{1,38}=4.713, p<.05\right.$, $\left.\eta^{2}=0.01\right)$. However, resolving the interaction again revealed that in both the isolated and transfer trials, touch points yielded more part-name learning than the $7 \mathrm{~cm}$ distance (isolated trials, touch: $\mathrm{M}=3.00, \mathrm{SE}=0.27 ; 7 \mathrm{~cm}: \mathrm{M}=0.55, \mathrm{SE}=0.20 ; p<.001$. transfer trials, touch: $\mathrm{M}=2.73, \mathrm{SE}=0.32 ; 7 \mathrm{~cm}: \mathrm{M}=0.90, \mathrm{SE}=0.22 ; p<.001)$. There was also a small interaction effect of Trial type and Motion $\left(F_{1,38}=4.577, p<.05, \eta^{2}=0.01\right)$. In the transfer trials, circular motion $(M=2.43, S E=0.33)$ yielded more part-name learning than still pointing $(M=1.29$, $\mathrm{SE}=0.32), p=.002$. In the isolated trials, this pattern was not statistically significant.

\section{Two-and-a-half-year-olds}

Regarding two-and-a-half-year-olds, a 2 (Distance) $\times 2$ (Motion) $\times 2$ (Trial type) ANOVA revealed main effects of Motion $\left(F_{1,31}=22.699, p<.001, \eta^{2}=0.323\right)$, with circular motion $(\mathrm{M}=2.41, \mathrm{SE}=0.24)$ leading to more part-name learning than still pointing $(\mathrm{M}=0.92$, $\mathrm{SE}=0.22)$. Distance produced a marginally significant effect $\left(F_{1,31}=2.917, p=.097\right.$, $\left.\eta^{2}=0.041\right)$, with slightly more part-name learning for touching $(\mathrm{M}=1.92, \mathrm{SE}=0.31)$ than $7 \mathrm{~cm}$ distance $(\mathrm{M}=1.35, \mathrm{SE}=0.27)$. No other effects or interactions were significant.

\subsection{Learning compared to chance}

Next, we compared part choices against chance to test whether participants would actually make more part choices than expected by chance. Visual inspection revealed that performance in both isolated and transfer trials was on the same side of chance levels, and previous analyses revealed no relevant effects of trial type; therefore, we averaged across trial types. One-sample t-tests with chance set to a value of 2 revealed that marked pointing through touch with circular motion yielded significant above-chance choice of the part object in each age group (all $t s>3.0, p s<.05$, two-tailed). For adults, the pattern was the same for touch points without circular motion. In contrast, four-and-a-half-yearolds hovered around chance level without the circular motion, and two-and-a-half-year- 
olds remained below chance without the circular motion $\left(t_{8}=2.712, p<.05\right.$, two-tailed). Distal pointing yielded part choices below chance across all age groups when the pointing was still (all $t \mathrm{~s}>3.0, \mathrm{ps}<.05$, two-tailed). Adding circular motion to distal pointing raised the youngest group to chance level.

\subsection{Analyses of switch responses across isolated trials and transfer trials}

The switch analyses were exploratory. Analyzing the difference score between choices in the isolated trials and transfer trials confirmed that participants did not systematically switch interpretations. In each age group, difference scores were significantly above chance in all conditions (all $t s>2.6, p s<.05$ ) except for two-and-a-half-year-olds in the still-and-touch condition $\left(t_{8}=1.793, p=.11\right)$. Two-and-a-half-year-olds were less consistent in their response in this condition.

\section{Discussion}

The current study revealed that marked pointing promotes learning of part names. The markedness of pointing was examined in regard to two aspects, distance and motion. Touch-pointing to the object part led adults to interpret a novel label as reference to the touched object part. Adding circular motion to the touch point also led the younger age groups to interpret the label as referring to the object part. In contrast, canonical pointing with a distance between the index finger and the object part, without markedness, led all age groups to interpret the label as referring to the whole object. In the youngest age group, adding a circular motion to the distal point loosened the whole-object interpretation to chance level. The pattern of findings reveals that the social pragmatic context of gestural reference promotes object-part name learning from the youngest age tested.

The stimulus objects, the target object parts, and the names for these items were all unfamiliar to the children. The assumption of mutual exclusivity requires familiarity with some object label in the task to constrain the interpretation space when learning a new word. Thus, by design, an interpretation of the results in terms of the constraint of mutual exclusivity can be excluded. In support, the pattern of findings was similar in isolated and transfer trials. What differed between conditions were the marking of the referential act through accompanying gestural features, implicating social-pragmatic factors in the process.

All participants predominantly interpreted the reference accompanied by the canonical still, distal, "unmarked" pointing to be referring to the whole object. One could argue that this result supports the assumption of the whole-object constraint: in the absence of marking, one could argue, participants revert to a default that the label must be referring to the whole object. Alternatively, a social-pragmatic account based on relevance assumptions (Sperber \& Wilson, 1995) would predict that the most parsimonious and effortless inference about the referential intention of the labeler in the distant still condition is the whole object - otherwise, if another detail was intended, the communicator needed to exhibit more effort in conveying a different relevance of the reference. While these two possibilities are difficult to tease apart in the current study when looking only at the still, distal pointing gesture, the pattern of all conditions clearly implicates social-pragmatic inferences. If young word learners used whole-object bias as a default bias, the youngest group's data will be distributed in a manner that shows their dependency on whole choices in all conditions. However, the youngest group's data already tended to vary according to conditions, as did those of other age groups. The account of relevance assumptions differs 
from that of a whole object constraint in that it holds that instead of a default interpretation of a whole object, the recipient makes an inference about the most relevant referent. Certainly, even two-and-a-half-year-olds and four-and-a-half-year-olds showed sensitivity to an adult's pointing gesture that was either touching a part or showing a small movement. When the touching and the movement were interpreted to be relevant in a certain situation for specifying the object part, such as the touch condition with motion, they chose part responses. When the movement was not interpreted to be relevant in a certain situation for specifying the object part, such as the distant pointing with motion, they chose whole responses. If this consideration is correct, this inference could also regard aspects other than the "wholeness" of an object. In support, studies that have manipulated other aspects of reference find that children flexibly apply a label either to an object or to an action, depending on the preceding shared action contexts (Akhtar et al., 1996).

The current findings also revealed differences in referential interpretation across age groups. All age groups predominantly chose the object part following touch-pointing with motion; however, only adults chose the object part following touch pointing without motion. On the first pass, children's preference to choose the whole object following touch pointing without motion may appear consistent with Markman's (1991) whole object assumption and Hansen and Markman's (2009) proposal that lexical constraints are more dominant than social-pragmatic cues. According to that account, current developmental findings would suggest that the constraint is lost across development. However, the results from the circular motion condition speak against a pure lexical constraint account and instead show that socio-pragmatic gestural information does influence the reference interpretation, even at the youngest age. A social-pragmatic interpretation of the age differences is thus that touch-pointing alone, without motion, does not sufficiently mark a distinct referent for the youngest age group. Young children may be very familiar with touch points from picturebook reading and other pedagogical scenarios with proximal toys and referents, in which a touch point is often used and accordingly interpreted to refer to a whole object. In contrast, in adult interactions, distal pointing is often a casual, effortless way of referring to global aspects of a scene or direction; thus, the effort of precisely touching one aspect clearly marks the referential intention in a distinct way (for a social-pragmatic distinction between "big" and "small" points, see also Enfield, Kita \& De Ruiter, 2007). Young children may initially be less familiar with the social-pragmatic marking of specific aspects through touch-pointing. Our results suggest that by approximately four and a half years old, children become more sensitive to this type of marking and equally adopt part and whole interpretations.

Human communication is inherently inferential (Grice, 1986; Sperber \& Wilson, 1995). To arrive at intended inferences, participants mark and pick up on relevant cues. Pedagogy theory (Csibra \& Gergely, 2009) has suggested that, at its base, ostensive cues mark actions as relevant "for you," that is, as communicative. Communicating episodic as opposed to generic information again requires specific semantic marking (Gelman \& Bloom, 2007), and in its absence, infants tend to interpret communicated information as generic (Gergely, Egyed \& Kiraly, 2007). Further studies have shown that infants and children infer the various reasons for communication, not only through common ground but also through distinct expressions and markedness of the act (Liebal et al., 2011; Liszkowski, 2014). One can argue that cues of markedness highlight and focus a learner's attention (Samuelson \& Smith, 1998). Indeed, when expressing an intended reference, the speaker's intention is to constrain the attentional focus of the recipient. Gogate, Maganti, and Laing (2013) showed that when caregivers intended to refer to a whole object, they held the object and shook it in front of the child's eyes. When they intended to refer to object parts, they tended to touch the object rather than simply holding and showing the 
object. Thus, expressing referential intentions inherently includes modifying the recipient's attention. However, social-pragmatic accounts would predict that the attentional focus is successfully constrained by inferences about the sender's intentions rather than by an endogenous process that directs attention. While the current study was not designed to tease apart these different predictions, other studies have shown that accidental (non-intended) actions, which do direct a recipient's attention, do not lead to the same referential inferences as intended actions (Diesendruck et al., 2004).

The current findings support the interpretation that word learning is governed by social-pragmatic inferences about a speaker's intention based on the way it is expressed through referential acts. This type of markedness is neither a fixed code nor part of constraints working under certain conditions. Instead, it develops through experience, as our developmental findings suggest. The expression of the relevance-of-reference - that is, the marking of reference in one way or another, accounts for word learning across diverse situations, including part-name learning in the absence of lexical constraints.

Acknowledgements. We would like to acknowledge all of the participants. We would also like to thank Editage (www.editage.jp) for English language editing. This study was partly supported by MEXT/the Japan Society for the Promotion of Science (JSPS) JP17H06382 (HK) and JSPS JP20H01763(HK) and JP20K03375 (TY).

\section{References}

Akhtar, N., Carpenter, M., \& Tomasello, M. (1996). The role of discourse novelty in early word learning. Child Development, 67(2), 635-645. doi:10.2307/1131837

Au, T. K. F., \& Glusman, M. (1990). The principle of mutual exclusivity in word learning: To honor or not to honor?. Child Development, 61(5), 1474-1490. doi:10.2307/1130757

Baldwin, D. A. (1991). Infants' contribution to the achievement of joint reference. Child Development, 62(5), 875-90. doi:10.2307/1131140

Behne, T., Liszkowski, U., Carpenter, M., \& Tomasello, M. (2012). Twelve-month-olds' comprehension and production of pointing. British Journal of Developmental Psychology, 30(3), 359-375. doi:10.1111/ j.2044-835X.2011.02043.x

Bloom, P. (2002). How children learn the meanings of words. Cambridge, MA: MIT press.

Bruner, J. S. (1981). Some elements of discovery. Thinking: The Journal of Philosophy for Children, 3(1), 26-31. doi:10.5840/thinking19813154

Carston, R. (2008). Thoughts and utterances: The pragmatics of explicit communication. John Wiley \& Sons.

Clark, E. V. (1997). Conceptual perspective and lexical choice in acquisition. Cognition, 64, 1-37. doi: 10.1016/S0010-0277(97)00010-3

Clark, E. V. (2009). First Language Acquisition (2nd edition). Cambridge, UK: Cambridge University Press.

Csibra, G., \& Gergely, G. (2009). Natural pedagogy. Trends in Cognitive Sciences, 13(4), 148-153. doi: 10.1016/j.tics.2009.01.005

Diesendruck, G., \& Markson, L. (2001). Children's avoidance of lexical overlap: A pragmatic account. Developmental Psychology, 37, 630-641. doi:10.1037/0012-1649.37.5.630

Diesendruck, G., Markson, L., Akhtar, N., \& Reudor, A. (2004). Two-year-olds' sensitivity to speakers' intent: An alternative account of Samuelson and Smith. Developmental Science, 7(1), 33-41. doi:10.1111/ j.1467-7687.2004.00320.x

Enfield, N. J., Kita, S., \& De Ruiter, J. P. (2007). Primary and secondary pragmatic functions of pointing gestures. Journal of Pragmatics, 39, 1722-1741. doi:10.1016/j.pragma.2007.03.001

Esteve-Gibert, N., Prieto, P., \& Liszkowski, U. (2017). Twelve-Month-Olds Understand Social Intentions Based on Prosody and Gesture Shape. Infancy, 22(1), 108-129. doi:10.1111/infa.12146

Gelman, S. A., \& Bloom, P. (2007). Developmental changes in the understanding of generics. Cognition, 105, 166-183. doi:10.1016/j.cognition.2006.09.009 
Gergely, G., Egyed, K., \& Kiraly, I. (2007). On pedagogy. Developmental Science, 10(1), 139-146. doi:10.111/ j.1467-7687.2007.00576.x

Gliga, T., \& Csibra, G. (2009). One-year-old infants appreciate the referential nature of deictic gestures and words. Psychological Science, 20(3), 347-353. doi:10.1111/j.1467-9280.2009.02295.x

Gogate, L. J., Maganti, M., \& Laing, K. B. (2013). Maternal naming of object wholes versus parts to preverbal infants: A fine-grained analysis of scaffolding at 6-8 months. Infant Behavior and Development, 36, 470-479. doi:10.1016/j.infbeh.2013.03.012

Gollek, C., \& Doherty, M. J. (2016). Metacognitive developments in word learning: Mutual exclusivity and theory of mind. Journal of Experimental Child Psychology, 148, 51-69. doi:10.1016/j.jecp.2016.03.007

Grassmann, S., Stracke, M., \& Tomasello, M. (2009). Two-year-olds exclude novel objects as potential referents of novel words based on pragmatics. Cognition, 112(3), 488-493. doi:10.1016/j.cognition.2009.06.010

Grice, H. P. (1986). Logic and conversation, University of California, Berkeley.

Grünloh, T., \& Liszkowski, U. (2015). Prelinguistic vocalizations distinguish pointing acts. Journal of Child Language, 42, 1312-1336. doi:10.1017/S0305000914000816

Hansen, M. B., \& Markman, E. M. (2009). Children's use of mutual exclusivity to learn labels for parts of objects. Developmental Psychology, 45, 592-596. doi:10.1037/a0014838

Jaswal, V. K. (2010). Explaining the disambiguation effect: Don't exclude mutual exclusivity. Journal of Child Language, 37(1), 95-113. doi:10.1017/S0305000909009519

Jaswal, V. K., \& Hansen, M. B. (2006). Learning words: Children disregard some pragmatic information that conflicts with mutual exclusivity. Developmental Science, 9(2), 158-165. doi:10.1111/j.1467-7687.2006.00475.x

Kalashnikova, M., Mattock, K., \& Monaghan, P. (2016). Flexible use of mutual exclusivity in word learning. Language Learning and Development, 12(1), 79-91. doi:10.1017/S1366728914000364

Kita, S. (2003). Pointing: A foundational building block of human communication. In S. Kita (ed.), Pointing: Where language, culture, and cognition meet (pp. 1-8). Mahwah, NJ: Erlbaum. doi:10.4324/9781410607744

Kobayashi, H. (1997). The role of actions in making inferences about the shape and material of solid objects among Japanese 2 year-old children. Cognition, 63, 251-269. doi:10.1016/S0010-0277(97)00007-3

Kobayashi, H. (1998). How 2-year-old children learn novel part names of unfamiliar objects. Cognition, 68, B41-B51. doi:10.1016/S0010-0277(98)00044-4

Kobayashi, H. (2002). Learning novel part names by observing adults' gestures. In Y. Shirai, H. Kobayashi, S. Miyata, K. Nakamura, T. Ogura, \& H. Sirai (eds.), Studies in Language Sciences 2 (pp. 149-156). Tokyo: Kurosio Publishers.

Kobayashi, H. (2007). The effect of touching object parts on learning novel object part names among young children and adults. In H. Sirai, S. Arita, M. Hirakawa, et al. (eds.), Studies in Language Science 6 (pp. 61 76). Tokyo: Kurosio Publishers.

Kobayashi, H., \& Yasuda, T. (2012). Meanings of touching object parts in pointing. In T. C. Scott-Phillips, M. Tamariz, E. A. Cartmill, \& J. R. Hurford (eds.), The Evolution of Language: Proceedings of the 9th International Conference on the Evolution of Language (pp. 181-188). Singapore: World Scientific. doi: 10.1142/9789814401500_0024

Liebal, K., Carpenter, M., \& Tomasello, M. (2011). Young children's understanding of markedness in nonverbal communication. Journal of Child Language, 38, 888-903. doi:10.1017/S0305000910000383

Liszkowski, U. (2014). Two sources of meaning in infant communication: preceding action contexts and actaccompanying characteristics. Philosophical Transactions of the Royal Society B: Biological Sciences, 369, 20130294. doi:10.1098/rstb.2013.0294

Liszkowski, U. (2018). Emergence of shared reference and shared minds in infancy. Current Opinion in Psychology, 23, 26-29. doi:10.1016/j.copsyc.2017.11.003

Markman, E. M. (1991). Categorization in Children: Problems of Induction. Cambridge, MA: MIT Press, Bradford Books. doi:10.7551/mitpress/1750.001.0001

Markman, E. M., \& Wachtel, G. A. (1988). Children's use of mutual exclusivity to constrain the meanings of words. Cognitive Psychology, 20, 120-157. doi:10.1016/0010-0285(88)90017-5

Merriman, W. E., Bowman, L. L., \& MacWhinney, B. (1989). The mutual exclusivity bias in children's word learning. Monographs of the Society for Research in Child Development, i-129. doi:10.2307/1166130

Mervis, C. B., Golinkoff, R. M., \& Bertrand, J. (1994). Two-year-olds readily learn multiple labels for the same basic-level category. Child Development, 65, 1163-1177. doi:10.1111/j.1467-8624.1994.tb00810.x

Moll, H., Koring, C., Carpenter, M., \& Tomasello, M. (2006). Infants determine others' focus of attention by pragmatics and exclusion. Journal of Cognition and Development, 7, 411-430. doi:10.1207/s15327647jcd0703_9 
Pinker, S. (2003). The language instinct: How the mind creates language. Penguin UK.

Quine, W., \& Van, O. (1960). Word and object: An inquiry into the linguistic mechanisms of objective reference. John Wiley.

Rüther, J., \& Liszkowski, U. (2020). Ontogenetic emergence of cognitive reference comprehension. Cognitive Science, 44(7), e12869. Doi:10.1111/cogs.12869

Samuelson, L. K., \& Smith, L. B. (1998). Memory and attention make smart word learning: An alternative account of Akhtar, Carpenter, and Tomasello. Child Development, 69(1), 94-104. doi:10.1111/j.14678624.1998.tb06136.x

Saylor, M. M., Sabbagh, M. A., \& Baldwin, D. A. (2002). Children use whole-part juxtaposition as a pragmatic cue to word meaning. Developmental Psychology, 38, 993-1003. doi:10.1037/0012-1649.38.6.993

Smith, L. B. (2001). How domain-general processes may create domain-specific biases. Language acquisition and conceptual development (pp. 101-131). Bowerman, M., Levinson, S. C., \& Levinson, S. (eds.) Language acquisition and conceptual development, Cambridge University Press. doi:10.1017/CBO9780511620669.006

Sperber, D., \& Wilson, D. (1995). Relevance: Communication and Cognition 2nd edition, Oxford: Blackwell. Tomasello, M. (2000). The social-pragmatic theory of word learning. Pragmatics, 10, 401-413. doi:10.1075/ prag.10.4.01tom

Tomasello, M. (2008). Origins of Human Communication. Cambridge, MA: MIT Press. doi:10.7551/ mitpress/7551.001.0001

Tomasello, M. (2009). Universal grammar is dead. Behavioral and Brain Sciences, 32(5), 470-471. doi: 10.1017/S0140525X09990744

Tomasello, M., \& Haberl, K. (2003). Understanding attention: 12-and 18-month-olds know what is new for other persons. Developmental Psychology, 39(5), 906. doi:10.1037/0012-1649.39.5.906

Yu, C., \& Smith, L. B. (2012). Embodied attention and word learning by toddlers. Cognition, 125(2), 244-262. doi:10.1016/j.cognition.2012.06.016

Cite this article: Kobayashi H., Yasuda T., \& Liszkowski U. (2023). Marked pointing facilitates learning part names: A test of lexical constraint versus social pragmatic accounts of word learning. Journal of Child Language 50, 296-310, https://doi.org/10.1017/S0305000921000891 